\title{
GMO analysis - a complex and challenging undertaking
}

\author{
Hendrik Emons
}

Published online: 28 January 2010

(C) Springer-Verlag 2010

Provide reliable analytical data for decisions! This demand has been, and is still, the main driver behind analytical developments in the area of genetically modified (GM) food or feed and related products of biotechnology. Therefore, many authors contributing to this special ABC issue refer to such regulatory requirements in the introductions of their papers. And, despite ongoing debates in the economic and public domains about the benefits and risks of the so-called green biotechnology, there is no doubt about the need to be able to investigate and monitor the presence, identity, and quantity of GM events in complex types of samples, from seeds and microorganisms to processed food.

One has to confess that after years of development and progress, the related analytical challenges are still manifold. They range from commonly acceptable sampling strategies via sufficiently described and understood sample-preparation procedures and efficient identification approaches to quantification methods which could deliver accurate data in a comparable fashion. Regulatory demands have pushed GM analysis from the qualitative to the quantitative level. Indeed, quantification of genetically modified constituents in food and feed is a relatively new area of chemical analysis or "bioanalysis". Basically two different types of analytes can be targeted for this purpose, namely the parts of the transgenic DNA which were inserted, with the help of biotechnological techniques, into the genome of a natural plant or the protein expressed by this transgenic DNA. Real-time polymerase chain reaction (rt-PCR) has

\section{H. Emons $(\bowtie)$}

European Commission, Joint Research Centre,

Institute for Reference Materials and Measurements (IRMM),

Retieseweg 111,

2440 Geel, Belgium

e-mail: hendrik.emons@ec.europa.eu become the technique widely used for quantification of GM constituents in food and feed and this is also documented in the current $\mathrm{ABC}$ issue. But its wider application in routine analysis should not ignore some of its principle limitations, which every method inherently has, and current methodical problems, which have still to be overcome, for example calibration and quality-assurance issues.

In the analysis of GMOs, interdisciplinary communication, education, and knowledge exchange are required for scientific understanding to progress. The combination of knowledge and techniques from plant sciences and molecular biology with principles and experimental methods of metrology and analytical chemistry creates the basis for new GM identification or quantification strategies and corresponding developments of instrumentation and procedures. Many of the authors of this special issue are working in such interdisciplinary teams and can enjoy the stimulating atmosphere of "out-of-the-box" thinking.

In recent years another characteristic of GMO analysis was the increased level of international harmonisation activities. This is not only evident in the work programs and published documents of standardisation and metrology organisations such as the ISO, Codex Alimentarius, or CIPM-CCQM, but also in the records of topics and speakers at international conferences and workshops on GMO analysis. The list of authors who contributed to this special issue is another piece of evidence of enhanced international cooperation.

The very positive response from many colleagues to the announcement of a special ABC issue on GMO analysis has enabled the compilation of an interesting collection of publications. Approaches for the first step of an analytical process, namely sampling, are discussed by Šuštar-Vozlič et al. New approaches for efficient screening for an increasing 
number of DNA inserts are discussed by Ruttink et al., von Goetz, Querci et al., Dörries et al., Lu et al., Waiblinger et al., Raymond et al., Bahrdt et al., Van den Bulcke et al., and Dobnik et al.. The detection targets include known (legally authorized or unauthorized) and unknown GM events and cover single and multiple genetic modification of the plant genome. A new road is paved by the development and use of microarrays for GM screening offering high-throughput (or, better, highly simultaneous) detection capabilities. Unambiguous identification of DNA fragments in the various varieties of modified plants is the topic of another contribution (La Paz et al.). But GMO analysis in food and feed must also take into account the effect of food processing on the subsequent DNA characterisation (Gryson) including the consequences for DNA pre-amplification (Del Gaudio et al.). Moreover, DNA extraction is often a crucial step in the whole analytical procedure of real samples (Demeke and Jenkins). A critical view on the various "chemistries" used in real-time PCR points to potential efficiency gains (Gašparič et al.). Székács et al. have measured the expression of proteins in the leaves of genetically modified maize.

Another group of papers in this issue highlights challenges and progress in the GMO quantification. Calibration issues are tackled by using dual-target plasmids (Lievens et al., Debode et al.) or are partially circumvented by exploiting the recently available digital PCR technique (Corbisier et al.). But, irrespective of the strategy and methods used, quantitative results have to be related to globally accepted reference measurement systems based on metrological principles. Their main components and features, for example measurement units and metrological traceability, are still not commonly established and implemented (Trapmann et al.). Moreover, sufficiently validated methods must be used for GMO quantification, as for any other analytical task which aims to provide data reliable enough for decision making. The survey of Holden et al. underlines this prerequisite, and Papazova et al. present an evaluation of currently used methods targeting endogenous sequences for maize. Scholtens et al. propose a potentially more efficient approach for corresponding method validation. Several papers in this issue provide evidence about the currently achieved comparability of analytical results and required improvements regarding their quality assurance.

This special ABC issue on GMO analysis could only be realised because of the enthusiasm and willingness of all contributing authors to submit their manuscripts into the usual critical peer-review process of this journal at a given time. Therefore, I would like to thank all of them and also the publishing team of Analytical and Bioanalytical Chemistry for fruitful cooperation.

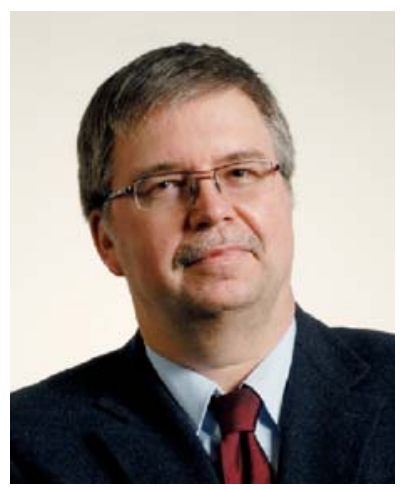

\section{Hendrik Emons}

is Head of the Reference Materials Unit of the European Commissions' Joint Research Centre, Institute for Reference Materials and Measurements (IRMM), located in Geel, Belgium. He is in charge of programmes on the development, production, and certification of reference materials for a very broad range of application areas from food and microbiological to environmental analysis and nuclear safeguards, from biotechnology (including GMOs) and clinical analysis to engineered materials and nanotechnology. He is, moreover, Associate Professor at the University of DuisburgEssen, Germany, and is on various scientific advisory boards and committees, for instance acting as Chairman of the ISO Committee on Reference Materials (REMCO) and member of the Research Council of the European Metrology Research Programme (EMRP) and the Executive Committee of AOAC's Technical Division on Reference Materials. He serves also on the Advisory Board of Analytical and Bioanalytical Chemistry. 